\title{
FIBRATIONS AND HOMOTOPY TYPE
}

\author{
J. L. NOAKES
}

\begin{abstract}
We prove the results announced in [13]. These allow us to distinguish homotopy types of spherical fibrations using fibre homotopy invariants.
\end{abstract}

Introduction. Let $E_{1}, E_{2}$ be Hurewicz fibrations over a connected finite $\mathrm{CW}$ complex $B$. We say that they are fibre homotopy equivalent $\left(E_{1} \simeq{ }_{B} E_{2}\right)$ when there are fibre preserving maps $\tau: E_{1} \rightarrow E_{2}, \gamma: E_{2} \rightarrow E_{1}$ such that $\gamma \tau, \tau \gamma$ are homotopic through fibre preserving maps to the identities on $E_{1}, E_{2}$. When this happens $\tau$ defines a homotopy equivalence $\left(E_{1}, F_{1}\right) \simeq\left(E_{2}, F_{2}\right)$ of pairs, where $F_{1}, F_{2}$ are the fibres over a basepoint of $B$. A result of A. Dold [2] asserts that $E_{1} \simeq_{B} E_{2}$ provided there is a fibre preserving map $\tau: E_{1} \rightarrow E_{2}$ whose restriction to fibres is a homotopy equivalence $F_{1} \simeq F_{2}$.

We take $F_{1}, F_{2}$ to be homotopy $q$-spheres $S_{1}^{q}, S_{2}^{q}(q \geqslant 1)$, and we try to relate the fibre homotopy types of $E_{1}, E_{2}$ when either $E_{1} \simeq E_{2}$ or $\left(E_{1}, S_{1}^{q}\right) \simeq\left(E_{2}, S_{2}^{q}\right)$. Results of I. M. James and J. H. C. Whitehead $[7,8]$ can be expressed in the following form.

THEOREM JW. Let $E_{1}, E_{2}$ be orthogonal $q$-sphere bundles over a sphere $S^{r+1}$ with $E_{1} \simeq E_{2}$. If $E_{2}$ has a cross-section then

(i) $E_{1}$ has a cross-section, and

(ii) there is a homotopy equivalence $f: S^{r+1} \rightarrow S^{r+1}$ such that $E_{1}, f^{*} E_{2}$ have the same fibre homotopy type.

Also, in [4], S. Y. Husseini proved

THEOREM H. Let $B$ be simply connected with $\operatorname{dim} B<q$ and suppose $\left(E_{1}, S_{1}^{q}\right) \simeq$ $\left(E_{2}, S_{2}^{q}\right)$. Then there is a homotopy equivalence $f: B \rightarrow B$ such that $E_{1}, f^{*} E_{2}$ have the same fibre homotopy type.

We wish to generalise these results to a situation where $B$ is as general a space as possible and $E_{1}, E_{2}$ are not necessarily fibre bundles.

Chapters I and II are directed towards proving the following conjecture of I. M. James.

Conjecture J. If $E_{1} \simeq B \times S^{q}$ then $E_{1}$ is fibre homotopy trivial.

We prove this when $B$ is nilpotent [1] and either $E_{1}$ has a cross-section (I. Proposition 2) or $\operatorname{dim} B<2 q$ (II. Corollary 1). When $B$ is a sphere or when $H^{q}(B ; \mathbf{Z})$ is finite, Conjecture $\mathrm{J}$ is proved in [6], together with other special cases. We

Received by the editors July 3, 1980 and, in revised form, July 22, 1983.

1980 Mathematics Subject Classification. Primary 55R05, 55R10, 55P10; Secondary 55P60.

(C)1984 American Mathematical Society $0002-9939 / 84 \$ 1.00+\$ .25$ per page 
make repeated use of the following result, which can be proved using the counting argument of $[6, \S 3]$.

THeOREM J. If $E_{1}, E_{2}$ are Z-orientable with $E_{1} \simeq E_{2}$, then if the Euler class $\chi\left(E_{2}\right)$ vanishes so does $\chi\left(E_{1}\right)$. (A similar result holds for the $\bmod p$ Euler classes, and also for the localizations of the fibrations $E_{1}, E_{2}$.)

Although we use counting arguments in Chapter I, in the spirit of [6 and 7], there are several new ingredients in our proofs. The first is the use of localization (suggested to me by Professor James in connection with Conjecture J) in Chapters I and II. The second is the notion of an exchange which appears in Chapter II. When a homotopy equivalence $\tau: E_{1} \rightarrow E_{2}$ looks like a fibre homotopy equivalence from the point of view of cohomology, there is no exchange; otherwise we say there is an exchange. The key point in the proof of II. Theorem 1 is that when there is an exchange $B$ is describable in terms of $E_{2} \mid B^{q-1}$. Another novelty, in Chapter III, is the use of a co- $H$-spacelike structure on a suspended fibration to replace a homotopy equivalence by a fibre homotopy equivalence. This replaces Husseini's loopspace argument and proves a stronger result.

Our results apply mainly in situations where both $E_{1}$ and $E_{2}$ have cross-sections (but see II. Theorem 1 for an important exception). There are many unanswered questions when either $E_{1}$ or $E_{2}$ does not have a cross-section, and for background we refer to [9]. For example, does the following conjecture hold?

CONJECTURE N. If $E_{1} \simeq E_{2}$, where $E_{2}$ has a cross-section, then $E_{1}$ has a cross-section.

According to Proposition 2 this would imply Conjecture $\mathrm{J}$ when $B$ is nilpotent.

Finally, a word of thanks: the present paper is a combination of three unpublished notes (corresponding to the three chapters), and the presentation has been substantially improved by the helpful comments of two anonymous referees. Also my original proof of III. Theorem 2(i) required $E_{2}$ to be $\mathbf{Z}$-orientable and $B$ to be nilpotent. I owe to a referee the observation that these hypotheses are unnecessary.

\section{Chapter I. COUNTING ARguments}

1. Statement of results. Let $p$ be a prime. When $B$ is nilpotent and $E_{j} \rightarrow B$ is Z-orientable $(j=1,2)$, we can form the localization $E_{j(p)} \rightarrow B_{(p)}$. We denote the projections of $E_{j}, E_{j(p)}$ by $\pi_{j}$.

Proposition 1. Let $B$ be nilpotent, and let $E_{2}$ be $\mathbf{Z}$-orientable. Suppose, for some $1 \leqslant i \leqslant q, E_{2(2)} \mid\left(B^{q+i}\right)_{(2)}$ has a cross-section and $E_{1} \simeq E_{2}$. If the ith Stiefel-Whitney class $w_{i}\left(E_{2}\right) \in H^{i}(B ; \mathbf{Z} /(2))$ vanishes then so does $w_{i}\left(E_{1}\right)$.

REMARKS. (i) This is proved by an extension of the method used to prove Theorem $\mathrm{J}$. If $E_{1}$ is known to be $\mathbf{Z}$-orientable then it suffices to assume $E_{1(2)} \simeq E_{2(2)}$ instead of $E_{1} \simeq E_{2}$.

(ii) A similar result holds at the odd primes. It is necessary to know that $E_{1}$ is Z-orientable and to replace the Stiefel-Whitney classes by the Wu classes [11, p. 229]. 
(iii) If $f: B \rightarrow B$ is a homotopy equivalence, then $\left(E_{2}, S_{2}^{q}\right) \simeq\left(f^{*} E_{2}, S_{2}^{q}\right)$, but $w_{i}\left(E_{2}\right) \neq w_{i}\left(f^{*} E_{2}\right)$ in general. For example, we may take $f: S^{2} \times S^{2} \rightarrow S^{2} \times S^{2}$ to be the switching map, and $E_{2}$ to be induced from the Hopf circle bundle over $S^{2}$ by projection to the second factor. We may fibre suspend twice to get an example with $\operatorname{dim} B<q$.

Proposition 2. Let $B$ be nilpotent and suppose $E_{1} \simeq B \times S^{q}$. If $E_{1(p)}$ has a cross-section for all primes $p$ then $E_{1}$ is fibre homotopy trivial.

This bears on Conjecture J. We have more to say in Chapter II.

EXAMPLES. (i) Let $E_{1}$ be the fibre suspension of the tangent sphere bundle of the complex projective plane $\mathrm{C} P^{2}$, and let $E_{2}$ be the fibre suspension of the fibre join of two copies of the Hopf circle bundle over $\mathbf{C} P^{2}$. Because both bundles have cross-sections their cohomology rings are isomorphic. However $E_{1} \neq E_{2}$. In effect Proposition 1 says that the homotopy types of $E_{1}, E_{2}$ are distinguished by the operation $\mathrm{Sq}^{2}$.

(ii) Suppose $E_{1} \simeq B \times S^{q}$ with $\operatorname{dim} B \leqslant q+1$. By Proposition 1, $E_{1}$ is Z-orientable, and so $E_{1}$ has a cross-section by Theorem $\mathrm{J}$. If $B$ is nilpotent then $E_{1}$ is fibre homotopy trivial by Proposition 2. This proves Conjecture $\mathbf{J}$ when $\operatorname{dim} B \leqslant q+1$ and $B$ is nilpotent, but we do better in Chapter II.

(iii) There are sphere bundles $E_{1} \neq E_{2}$ over a sphere, and a finite CW complex $A$, such that $A \times E_{1} \simeq A \times E_{2}$ [3]. Proposition 2 with [6, 2.2] shows that this cannot happen when $E_{2}$ is trivial.

2. Characteristic classes. In the context of Proposition 1 let $s_{2}^{\prime}$ be a cross-section of $E_{2(2)} \mid\left(B^{q+i}\right)_{(2)}$ and let $\pi_{2}^{\prime}$ be the restriction of $\pi_{2}$ to $E_{2(2)} \mid\left(B^{q+i}\right)_{(2)}$. We denote the inclusions $\left(B^{q+i}\right)_{(2)} \rightarrow B_{(2)}, E_{2(2)} \mid\left(B^{q+i}\right)_{(2)} \rightarrow E_{2(2)}$ by $\iota^{\prime}$. Then the $\mathbf{Z} /(2)$-Gysin sequences give us the following commuting diagrams with exact rows $(r \geqslant 0)$.

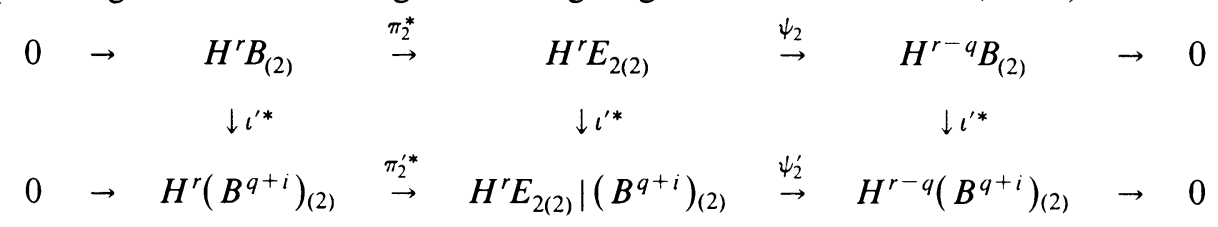

Let $l \in H^{0}\left(B^{q+i}\right)_{(2)}$ be the generator and let $a_{0} \in H^{q}\left(E_{2(2)} \mid\left(B^{q+i}\right)_{(2)}\right)$ satisfy $\psi_{2}^{\prime} a_{0}=1$. If $a_{2}^{\prime}=a_{0}-\pi_{2}^{\prime *} s_{2}^{\prime *} a_{0}$ then $\psi_{2}^{\prime} a_{2}^{\prime}=1$ and $s_{2}^{\prime *} a_{2}^{\prime}=0$.

Now $\iota^{\prime *}: H^{r} E_{2(2)} \rightarrow H^{r}\left(E_{2(2)} \mid\left(B^{q+i}\right)_{(2)}\right)$ is surjective for $r<q+i$, and we let $a_{2} \in H^{q} E_{2(2)}$ satisfy $\iota^{\prime *} a_{2}=a_{2}^{\prime}$. Then $\psi_{2} a_{2}=1$ so $\psi_{2} \mathrm{Sq}^{i} a_{2}$ is the localization of $w_{i}\left(E_{2}\right)$. But $w_{i}\left(E_{2}\right)=0$ by hypothesis, so $\mathrm{Sq}^{i} a_{2}=\pi_{2}^{*} b$ for some $b \in H^{q+i} B_{(2)}$. Therefore

$$
\mathrm{Sq}^{i} a_{2}^{\prime}=\iota^{\prime *} \mathrm{Sq}^{i} a_{2}=\iota^{\prime *} \pi_{2}^{*} b=\pi_{2}^{\prime *} \iota^{\prime *} b
$$

so we have

$$
\iota^{\prime *} b=s_{2}^{\prime *} \pi_{2}^{\prime *} \iota^{\prime *} b=s_{2}^{\prime *} \mathrm{Sq}^{i} a_{2}^{\prime}=\mathrm{Sq}^{i} s_{2}^{\prime *} a_{2}^{\prime}=0 .
$$

Therefore $b=0$ because $\iota^{\prime *}: H^{r} B_{(2)} \rightarrow H^{r}\left(B^{q+i}\right)_{(2)}$ is injective for $r \leqslant q+i$. It follows that $\mathrm{Sq}^{i} a_{2}=0$ so

$$
\mathrm{Sq}^{i}\left(H^{q} E_{2(2)}\right)=\pi_{2}^{*} \operatorname{Sq}^{i}\left(H^{q} B_{(2)}\right) .
$$


For a general CW complex $X$ let $\#(i) X$ be the order of the subgroup $\operatorname{Sq}^{i}\left(H^{q} X\right)$ of $H^{q+i} X$. We note that if $X$ is nilpotent then $\#(i) X=\#(i) X_{(2)}$. Then $\#(i) E_{2}$, $\#(i) B<\infty$, and we know that $\#(i) E_{2}=\#(i) E_{2(2)}=\#(i) B_{(2)}=\#(i) B$. By Theorem $\mathrm{J}, w_{q+1}\left(E_{1}\right)=0$ because $w_{q+1}\left(E_{2}\right)=0$, so the $\mathbf{Z} /(2)=$ Gysin sequence of $E_{1}$ is

$$
0 \rightarrow H^{r} B \stackrel{\pi_{1}^{*}}{\rightarrow} H^{r} E_{1} \stackrel{\psi_{1}}{\rightarrow} H^{r-q} B \rightarrow 0
$$

for $r \geqslant 0$. Let $a_{1} \in H^{q} E_{1}$ satisfy $\psi_{1} a_{1} \neq 0$. Then $w_{i}\left(E_{1}\right)=\psi_{1} \mathrm{Sq}^{i} a_{1}=0$ since $\#(i) E_{1}=\#(i) E_{2}=\#(i) B$. This proves Proposition 1 .

3. Decomposability. If $E_{1} \simeq B \times S^{q}$ then, by Proposition 1, $E_{1}$ is Z-orientable. We observe that if $E_{1(p)}$ is fibre homotopy trivial for all primes $p$ then $E_{1}$ is fibre homotopy trivial. One way of seeing this is to note that a fibre homotopy equivalence corresponds as in $[14, \S 1]$ to a cross-section of a function space fibration. We may then use $[15,3.2]$.

We denote the inclusion of the fibre in $E_{1(p)}$ by $\iota_{1}$. Then we have an exact sequence

$$
0 \rightarrow H^{q} B_{(p)} \stackrel{\pi_{1}^{*}}{\rightarrow} H^{q} E_{1(p)} \stackrel{\stackrel{\iota^{*}}{\rightarrow}}{\rightarrow} H^{q} S_{(p)}^{q} \rightarrow 0
$$

with coefficients $\mathbf{Z} /(p)$. For a general CW complex $X$ we call $x \in H^{q} X$ cospherical when it lies in the image of $f^{*}: H^{q} S_{(p)}^{q} \rightarrow H^{q} X$ for some $f: X \rightarrow S_{(p)}^{q}$. Let $\# X$ be the number of cospherical elements of $H^{q} X$.

Then $\# E_{1(p)}, \# B_{(p)}<\infty$ and $\# E_{1(p)}=\#\left(B \times S^{q}\right)_{(p)} \geqslant\left(\# B_{(p)}\right)+1$. So some cospherical $a \in H^{q} E_{1(p)}$ is not $\pi_{1}^{*} c$ for any cospherical $c \in H^{q} B_{(p)}$. Now $\iota_{1}^{*} a \neq 0$ because otherwise we would have $a=\pi_{1}^{*} b$, and $b$ would have to be cospherical (it is here that we use the hypothesis that $E_{1(p)}$ has a cross-section). So for some $f: E_{1(p)} \rightarrow S_{(p)}^{q}$ the composite $f_{\iota_{1}}: S_{1(p)}^{q} \rightarrow S_{(p)}^{q}$ is a homotopy equivalence (here we see the usefulness of localizing at $p)$. So $E_{1(p)}$ is fibre homotopy trivial, and this proves Proposition 2.

\section{Chapter II. Cross-Sections}

1. Statement of results. The proof of Theorem JW(i) uses a counting argument and the key point is that $G=\pi_{r} S_{l}^{q}$ satisfies the endomorphism condition, namely the surjective endomorphisms of $G$ are isomorphisms. It suffices for $G$ to be finitely generated, and so in [7] the fibres of $E_{1}, E_{2}$ are not required to be spheres.

In our situation $B$ is no longer a suspended space, and the counting argument of [7] is not available. However, taking $p$ to be a prime, we have the following $p$-local result.

Theorem 1. Let $B$ be nilpotent, and let $E_{1}, E_{2}$ be Z-orientable with $\operatorname{dim} B<2 q$. Suppose $E_{2}$ has a cross-section and $E_{1(p)} \simeq E_{2(p)}$ where $p$ is prime. Then $E_{1(p)}$ has a cross-section. 
Comparing this with I. Proposition 2 we obtain

Corollary 1. Let $B$ be nilpotent with $\operatorname{dim} B<2 q$. If $E_{1} \simeq B \times S^{q}$ then $E_{1}$ is fibre homotopy trivial.

So we have another special case of Conjecture J. Theorem 1 appears to support Conjecture $\mathrm{N}$, although it seems to be unknown whether $E_{1}$ has a cross-section when $E_{\mathrm{l}(p)}$ has a cross-section for all primes $p$. (There is no difficulty when $B$ is a sphere, or when $H^{q}(B ; Q)=0$, or, less trivially, when $q$ is odd.) Interestingly, the endomorphism condition for finitely generated $\mathbf{Z}_{(p)}$-modules plays a part in our proof of Theorem 1.

2. No exchange. Let $s_{2}$ be a cross-section of $E_{2(p)}$ and let $\tau: E_{1(p)} \rightarrow E_{2(p)}$ be a homotopy equivalence with homotopy inverse $\gamma$. By Theorem $\mathrm{J}$ we have $\chi\left(E_{1(p)}\right)=0$ because $\chi\left(E_{2(p)}\right)=0$. This proves Theorem 1 when $q=1$, and we exclude this case for the remainder of Chapter II. The Gysin sequences give us the diagrams

$$
\begin{aligned}
0 \rightarrow H^{r} B_{(p)} \stackrel{\pi_{1}^{*}}{\rightarrow} H^{r} E_{1}(p) \stackrel{\psi_{1}}{\rightarrow} H^{r-q} B_{(p)} \rightarrow 0 \\
\gamma^{*} \downarrow \uparrow \tau^{*} \\
0 \rightarrow H^{r} B_{(p)} \stackrel{\pi_{2}^{*}}{\rightarrow} H^{r} E_{2(p)} \stackrel{\psi_{2}}{\rightarrow} H^{r-q} B_{(p)} \rightarrow 0
\end{aligned}
$$

with exact rows $(r \geqslant 0)$. Here we take coefficients in the ring $\mathbf{Z}_{(p)}$ of integers localized at $p$. Proceeding as in I, $\S 2$, let $1 \in H^{0} B_{(p)}$ be the identity and let $a_{0} \in H^{q} E_{2(p)}$ satisfy $\psi_{2} a_{0}=1$. Let $a_{2}=a_{0}-\pi_{2}^{*} s_{2}^{*} a_{0}$. Then $\psi_{2} a_{2}=1$ and $s_{2}^{*} a_{2}=0$. We say there is no exchange when $\psi_{1} \tau^{*} a_{2}$ is a unit of $H^{0} B_{(p)}$. For the remainder of $\$ 2$ we assume this is the case, and we let $a_{1}=\tau^{*} a_{2}$.

We note that, for $r<q, s_{2}^{*} \gamma^{*} \pi_{1}^{*}: H^{r} B_{(p)} \rightarrow H^{r} B_{(p)}$ is a $\mathbf{Z}_{(p)}$-module automorphism. For $r \geqslant q$ let $b \in H^{r} B_{(p)}$ and, as in [10], write $\tau^{*} \pi_{2}^{*} b$ in the form

$$
\pi_{1}^{*} c+a_{1} \cdot \pi_{1}^{*} d
$$

where $c \in H^{r} B_{(p)}, d \in H^{r-q} B_{(p)}$. Then $b$ is

$$
s_{2}^{*} \gamma^{*}\left(\pi_{1}^{*} c+a_{1} \cdot \pi_{1}^{*} d\right)=s_{2}^{*} \gamma^{*} \pi_{1}^{*} c+\left(s_{2}^{*} a_{2}\right) \cdot\left(s_{2}^{*} \gamma^{*} \pi_{1}^{*} d\right)
$$

Since $s_{2}^{*} a_{2}=0$, we have $b=s_{2}^{*} \gamma^{*} \pi_{1}^{*} c$, so the $\mathbf{Z}_{(p)}$-module endomorphism $s_{2}^{*} \gamma^{*} \pi_{1}^{*}$ of $H^{r} B_{(p)}$ is surjective. But $H^{r} B_{(p)}$ is a finitely generated $\mathbf{Z}_{(p)}$-module, so $s_{2}^{*} \gamma^{*} \pi_{1}^{*}$ is an automorphism for all $r$.

So $\pi_{1} \gamma s_{2}: B_{(p)} \rightarrow B_{(p)}$ is a homotopy equivalence. Let $\beta$ be a homotopy inverse. Then $\gamma s_{2} \beta$ is homotopic to a cross-section of $E_{1(p)}$, and this proves Theorem 1 when there is no exchange.

3. Exchange. We treat the case where $\psi_{1} \iota^{*} a_{2}$ is not a unit in $\mathbf{Z}_{(p)}$. We say there is an exchange, and it is here that we use the hypothesis $\operatorname{dim} B<2 q$. We denote the 
inclusions $\left(B^{q-1}\right)_{(p)} \rightarrow B_{(p)}, E_{2(p)} \mid\left(B^{q-1}\right)_{(p)} \rightarrow E_{2(p)}$ by $\iota$. The Gysin sequences give us the commuting diagrams
$0 \quad \rightarrow \quad H^{r} B_{(p)} \quad \stackrel{\pi_{1}^{*}}{\rightarrow}$
$H^{r} E_{1(p)}$
$\stackrel{\psi_{1}}{\rightarrow}$
$H^{r-q} B_{(p)}$
$0 \rightarrow H^{r} B_{(p)}$
$\gamma^{*} \downarrow \uparrow \tau^{*}$
$\downarrow \iota^{*}$
$H^{r} E_{2(p)}$
$\stackrel{\psi_{2}}{\rightarrow}$
$H^{r-q} B_{(p)} \quad \rightarrow \quad 0$
$\iota^{*}$
$\downarrow$ เ*
$0 \rightarrow H^{r}\left(B^{q-1}\right)_{(p)} \stackrel{\pi_{2}^{\prime *}}{\rightarrow} H^{r} E_{2(p)} \mid\left(B^{q-1}\right)_{(p)} \stackrel{\psi_{2}^{\prime}}{\rightarrow} H^{r-q}\left(B^{q-1}\right)_{(p)} \quad \rightarrow \quad 0$

with exact rows $(r \geqslant 0)$. Here $\pi_{2}^{\prime}$ denotes the restriction of $\pi_{2}$, and we take coefficients in either of the rings $\mathbf{Z}_{(p)}$ or $\mathbf{Z}_{(p)} /(p) \cong \mathbf{Z} /(p)$.

With coefficients $\mathbf{Z}_{(p)} /(p)$ we have $\psi_{1} \tau^{*} a_{2}=0$ so $\tau^{*} a_{2}=\pi_{1}^{*} b_{1}$ for some $b_{1} \in$ $H^{q} B_{(p)}$. Let $\mathscr{B}$ be the Bockstein associated with the exact sequence

$$
0 \rightarrow \mathbf{Z}_{(p)} \stackrel{p}{\rightarrow} \mathbf{Z}_{(p)} \rightarrow \mathbf{Z}_{(p)} /(p) \rightarrow 0 .
$$

Then $\Re a_{2}=0$, so $0=\tau^{*} \Re a_{2}=\Re \tau^{*} a_{2}=\Re \pi_{1}^{*} b_{1}=\pi_{1}^{*} \Re b_{1}$. Therefore $\Re b_{1}=0$ so $b_{1}$ is the reduction of a $\mathbf{Z}_{(p)}$-cohomology class. Hence,

LEMMA 1. The homomorphism

$$
\iota^{*} \gamma^{*} \pi_{1}^{*}: H^{q}\left(B_{(p)} ; \mathbf{Z}_{(p)}\right) \rightarrow H^{q}\left(E_{2(p)} \mid\left(B^{q-1}\right)_{(p)} ; \mathbf{Z}_{(p)}\right)
$$

is surjective.

Working with coefficients $\mathbf{Z}_{(p)} /(p)$, we suppose $\iota^{*} \gamma^{*} \pi_{1}^{*} b=0$ where $b \in H^{r} B_{(p)}$ and $r \geqslant q$. Since $\operatorname{dim} B<2 q$, either $b=0$ or $r<2 q$. If $r<2 q$ then $\iota^{*}: H^{r-q} B_{(p)} \rightarrow$ $H^{r-q}\left(B^{q-1}\right)_{(p)}$ is injective. But $0=\psi_{2}^{\prime} \iota^{*} \gamma^{*} \pi_{1}^{*} b=\iota^{*} \psi_{2} \gamma^{*} \pi_{1}^{*} b$, so $\gamma^{*} \pi_{1}^{*} b=\pi_{2}^{*} c$ for some $c \in H^{r} B_{(p)}$. Now $\tau^{*}\left(a_{2} \cdot \pi_{2}^{*} c\right)=\left(\pi_{1}^{*} b_{1}\right)\left(\pi_{1}^{*} b\right)=0$ since $\operatorname{dim} B<2 q$ so $a_{2}$. $\pi_{2}^{*} c=0$. Applying $\psi_{2}$ to this equation we find that $c=0$. So $\gamma^{*} \pi_{1}^{*} b=0$ and $b=0$. This proves

Lemma 2. For $r \geqslant q$ the homomorphism $\iota^{*} \gamma^{*} \pi_{1}^{*}: H^{r} B_{(p)} \rightarrow H^{r}\left(E_{2(p)} \mid\left(B^{q-1}\right)_{(p)}\right)$ (with coefficients $\mathbf{Z}_{(p)} /(p)$ ) is injective.

Still working with coefficients $\mathbf{Z}_{(p)} /(p)$, we observe that since $\psi_{1} \tau^{*} a_{2}=0$ we have $\psi_{1} \tau^{*} \pi_{2}^{*} b_{2} \neq 0$ for some $b_{2} \in H^{q} B_{(p)}$. Let $a_{1}=\tau^{*} \pi_{2}^{*} b_{2}$. If $x \in H^{r} E_{2(p)}$, where $q \leqslant r<2 q-1$, we write $\tau^{*} x$ in the form $\pi_{1}^{*} c+a_{1} \cdot \pi_{1}^{*} d$, where $c \in H^{r} B_{(p)}, d \in$ $H^{r-q} B_{(p)}$. Then

$$
\iota^{*} x=\iota^{*} \gamma^{*}\left(\pi_{1}^{*} c+a_{1} \cdot \pi_{1}^{*} d\right)
$$

which we write as

$$
\iota^{*} \gamma^{*} \pi_{1}^{*} c+\iota^{*}\left(\left(\pi_{2}^{*} b_{2}\right) \cdot\left(\gamma^{*} \pi_{1}^{*} d\right)\right)
$$

Since $r<2 q-1<2 q$, we have $\gamma^{*} \pi_{1}^{*} d=\pi_{2}^{*} e$ for some $e \in H^{r-q} B_{(p)}$. So

$$
\iota^{*} x=\iota^{*} \gamma^{*} \pi_{1}^{*} c+\iota^{*}\left(\pi_{2}^{*}(b \cdot e)\right)=\iota^{*} \gamma^{*} \pi_{1}^{*} c+\pi_{2}^{\prime *} \iota^{*}\left(b_{2} \cdot e\right)=\iota^{*} \gamma^{*} \pi_{1}^{*} c
$$


because $H^{r}\left(B^{q-1}\right)_{(p)}=0$. But $\iota^{*}: H^{r} E_{2(p)} \rightarrow H^{r} E_{2(p)} \mid\left(B^{q-1}\right)_{(p)}$ is surjective for $q \leqslant r<2 q-1$, and this proves

LEMMA 3. For $q \leqslant r<2 q-1$ the homomorphism

$$
\iota^{*} \gamma^{*} \pi_{1}^{*}: H^{r} B_{(p)} \rightarrow H^{r}\left(E_{2(p)} \mid\left(B^{q-1}\right)_{(p)}\right)
$$

(with coefficients $\mathbf{Z}_{(p)} /(p)$ ) is surjective.

We form the induced fibrations $\pi_{1}^{*} E_{1(p)}, E=\iota^{*} \gamma^{*} \pi_{1}^{*} E_{1(p)}$ over $E_{1(p)}, E_{2(p)} \mid$ $\left(B^{q-1}\right)_{(p)}$.

$$
\begin{array}{ccccc}
E & \rightarrow & \pi_{1}^{*} E_{1(p)} & \rightarrow & E_{1(p)} \\
s \hat{\vdots} & & \downarrow & & \downarrow \\
E_{2(p)} \mid\left(B^{q-1}\right)_{(p)} & \stackrel{\gamma \iota}{\rightarrow} & E_{1(p)} & \stackrel{\pi_{1}}{\rightarrow} & B_{(p)}
\end{array}
$$

Then $\pi_{1}^{*} E_{1(p)}$ has a cross-section so $E$ has a cross-section $s$. But $\chi\left(E_{1(p)}\right)=0$, and obstruction theory with Lemmas 1-3 shows that $s$ gives rise to a cross-section of $E_{1(p)}$. This completes the proof of Theorem 1 .

\section{ChaPTER III. RElative HOMOTOPY TYPES}

1. Statement of results. In this chapter we are concerned with the situation when $E_{2}$ is a fibre suspension $\sum E$ for some $E \rightarrow B$. This implies $E_{2}$ has a cross-section, and some othe remarks about this condition are made in $\$ 2$.

THEOREM 2. Suppose $E_{2}$ is a fibre suspension and either

(i) $\left(E_{1}, S_{1}^{q}\right) \simeq\left(E_{2}, S_{2}^{q}\right)$, or

(ii) $E_{1} \simeq E_{2}$ with $H^{q}(B ; \mathbf{Z})$ finite, $E_{2} \mathbf{Z}$-orientable, and $B$ nilpotent.

Then there is a homotopy equivalence $f: B \rightarrow B$ such that $E_{1}, f^{*} E_{2}$ have the same fibre homotopy type.

REMARKS. (i) We see in $\S 2$ that if $\operatorname{dim} B<q$, then $E_{2}$ is automatically a fibre suspension. Consequently, Theorem 2 improves Theorem H. Note that [4, Theorem 1.1] is incorrectly stated: this is shown by I, $\S 1$, Remark (iii).

(ii) Taking $B=S^{r+1}$ in Theorem 2 we obtain Theorem JW except for the case where $r=q-1$. We deal with this case using our methods in the Appendix. Because we treat general spherical fibrations, and not just orthogonal sphere bundles, we can add to the picture in [8] with the following result.

COROllaRY 2. Let $\Phi \subseteq \pi_{r+q} S^{q}(0<r<q-1)$ be maximal with respect to the property that if $\alpha, \beta \in \Phi$ satisfy $\alpha+\beta=0$ then $\alpha=\beta$. Let $\phi$ be the evident function from $\Phi$ onto the set of homotopy types of q-spherical fibrations over $S^{r+1}$. Then $\phi$ is a bijection.

EXAMPLE. Let $\alpha$ generate $\pi_{8} S^{5} \cong \mathbf{Z} /(24)$ and let $E_{1}, E_{2}$ be the fibrations with fibre $S^{5}$ over $S^{4}$ classified by $5 \alpha, 11 \alpha$. Then for each prime $p$ there is a homotopy equivalence $f: S_{(p)}^{4} \rightarrow S_{(p)}^{(4)}$ such that $E_{1(p)}, f^{*} E_{2(p)}$ have the same fibre homotopy type. In particular, $E_{1}, E_{2}$ have the same genus [12]. But $E_{1} \approx E_{2}$. 
2. Desuspension. Let $G_{1}^{q}$ be the $H$-space of maps $S^{q} \rightarrow S^{q}$ of degree 1 with multiplication given by composition. Then, according to [17], there is a $q$-spherical fibration $U^{q}$ over the classifying space $B G_{1}^{q}$ with the following property. For any $\mathrm{CW}$ complex $X$ the correpondence $f \rightarrow f^{*} U^{q}$ defines a bijection from homotopy classes of maps $X \rightarrow B G_{1}^{q}$ to degree 1 fibre homotopy types of $q$-spherical fibrations over $X$. In particular, the fibre suspension $\Sigma U^{q-1}$ is induced by a map $g: B G_{1}^{q-1} \rightarrow B G_{1}^{q}$, and degree 1 fibre homotopy classes of $q-1$ spherical fibrations over $X$ which fibre suspend to $f^{*} U^{q}$ correspond bijectively to factorisations up to homotopy of $f$ through $g$. But a calculation using [18] shows that $g_{*}: \pi_{r} B G_{1}^{q-1} \rightarrow \pi_{r} B G_{1}^{q}$ is an isomorphism for $r<q-1$ and an epimorphism for $r=q-1$. This proves

Proposition 3. If $\operatorname{dim} B<q$, then $E_{2}$ is fibre homotopy equivalent to the fibre suspension of $a(q-1)$-spherical fibration $E$.

We next prove

Proposition 4. If $E_{2}$ is a q-sphere bundle (not necessarily orthogonal) with a cross-section, then $E_{2} \simeq_{B} \Sigma E$ for some bundle $E$.

We choose $s_{2}$ to map a basepoint of $B$ to $(0, \ldots, 1)$ in the fibre $S_{2}^{q}$ of $E_{2}$. The proof of [16, Lemma 1] shows that $E_{2}^{\prime}=E_{2}-s_{2}(B)$ is a fibre bundle. Since the fibre of $E_{2}^{\prime}$ is contractible, there is a cross-section $s_{2}^{\prime}$ of $E_{2}^{\prime}$. We choose $s_{2}^{\prime}$ to map the basepoint to $(0, \ldots,-1) \in S_{2}^{q}$. Then again, $E=E_{2}^{\prime}-s_{2}^{\prime}(B)$ is a fibre bundle.

We define the fibre suspension $\Sigma E$ in the usual way as a quotient of $E \times[-1,1]$. Now define $T: s_{2}(B) \cup s_{2}^{\prime}(B) \cup S_{2}^{q} \rightarrow[-1,1]$ by $T(e)=1,-1$, or $x_{q}$ according as $e \in s_{2}(B), e \in s_{2}^{\prime}(B)$, or $e=\left(x_{0}, \ldots, x_{q}\right) \in S_{2}^{q}$. Extending $T$ to a map $T: E_{2} \rightarrow$ $[-1,1]$, we define a fibre preserving map $h: E_{2} \rightarrow \sum E$ by $h(e)=\left[e, T_{2}(e)\right]$ for $e \in E$. We define $h(e)$ to be $\left[e^{\prime},-1\right]$ for $e \in s_{2}(B), e \in s_{2}^{\prime}(B)$, where $\pi_{2}\left(e^{\prime}\right)=\pi_{2}(e)$ and $e^{\prime} \in E$. Then it is easy to check that $h$ restricts to a homotopy equivalence on fibres. This proves Proposition 4.

3. No exchange. We argue from the situation of Theorem 2, except that we do not yet require $E_{2}$ to be a fibre suspension: it suffices to suppose that $E_{2}$ has a cross-section $s_{2}$. Let $\tau: E_{1} \rightarrow E_{2}$ be a homotopy equivalence with homotopy inverse $\gamma$ (in the case of Theorem 2(ii) we take $\tau, \gamma$ to be homotopy equivalences of pairs). Let $\iota_{j}: S_{j}^{q} \rightarrow E_{j}$ be the inclusions.

LEMma. The composite $\pi_{1} \gamma s_{2}: B \rightarrow B$ is a homotopy equivalence.

To prove this in the case of Theorem 2(i) we have the commuting diagrams $(r \geqslant 0)$

$$
\begin{array}{ccc}
\pi_{r}\left(E_{2}, S_{2}^{q}\right) & \stackrel{\gamma}{\rightrightarrows} & \pi_{r}\left(E_{1}, S_{1}^{q}\right) \\
\pi_{2_{*} \downarrow} \downarrow \| \uparrow s_{2_{*}} & & \| l \downarrow \pi_{1_{*}} \\
\pi_{r}(B, \cdot) & \stackrel{\left(\pi_{1} \gamma s_{2}\right)_{*}}{\rightarrow} & \pi_{r}(B, \cdot)
\end{array}
$$

from which it follows that the $\left(\pi_{1} \gamma s_{2}\right)_{*}$ are isomorphisms for all $r$. In Case (ii) we refer to the argument of II, $\S 2$, and this completes the proof. 
We now require $E_{2}$ to be $\Sigma E$ and let $s_{2}$ be the cross-section given by $s_{2}(b)=[e, 1]$ where $\pi_{2}(e)=b$. We define a fibre preserving map $\rho: \sum E \rightarrow \sum E$ by $\rho[e, t]=[e,-t]$. Then $\rho s_{2}$ is another cross-section of $E_{2}$, and $\pi_{1} \gamma \rho s_{2}$ is a homotopy equivalence by the preceding lemma. Let $\beta$ be a homotopy inverse. Then $\gamma \rho s_{2} \beta$ is homotopic to a cross-section $s_{1}$ of $E_{1}$ and $s_{1}\left(\pi_{1} \gamma \rho s_{2}\right) \simeq \gamma \rho s_{2}$. We note that $s_{2}$ is a cofibration, and so we can choose $\gamma^{\prime} \simeq \gamma \rho$ so that the following diagram commutes.

$$
\begin{array}{ccc}
B & \stackrel{\pi_{1} \gamma \rho s_{2}}{\rightarrow} & B \\
s_{2} \downarrow & & \downarrow s_{1} \\
\Sigma E & \stackrel{\gamma^{\prime}}{\rightarrow} & E_{1}
\end{array}
$$

Given maps $\theta_{1}, \theta_{2}, \theta_{3}: \Sigma E \rightarrow X$ satisfying $\theta_{1} s_{2}=\theta_{2} \rho s_{2}, \theta_{2} s_{2}=\theta_{3} \rho s_{2}$, we define a map $*\left(\theta_{1}, \theta_{2}, \theta_{3}\right) ; \sum E \rightarrow X$ by

$$
\begin{aligned}
{[e, t] } & \mapsto \theta_{1}[e, 3 t+2], & & -1 \leqslant t \leqslant-1 / 3, \\
& \mapsto \theta_{2}[e, 3 t], & & -1 / 3 \leqslant t \leqslant 1 / 3, \\
& \mapsto \theta_{3}[e, 3 t-2], & & 1 / 3 \leqslant t \leqslant 1 .
\end{aligned}
$$

We choose $X=E_{1}$ and $\theta_{1}=s_{1} \pi_{1} \gamma^{\prime}, \theta_{2}=s_{1} \pi_{1} \gamma \rho s_{2} \pi_{2}, \theta_{3}=\gamma^{\prime} \rho$. Then the necessary equations are satisfied, and we form $\theta=*\left(\theta_{1}, \theta_{2}, \theta_{3}\right): \Sigma E \rightarrow E_{1}$. Now $\pi_{1} \theta$ $=*\left(\pi_{1} \gamma^{\prime}, \pi_{1} \gamma \rho s_{2} \pi_{2}, \pi_{1} \gamma^{\prime} \rho\right): \sum E \rightarrow B$ and we note that the restriction of $\pi_{1} \gamma \rho s_{2} \pi_{2}$ to the fibre over any given point $b \in B$ is constant.
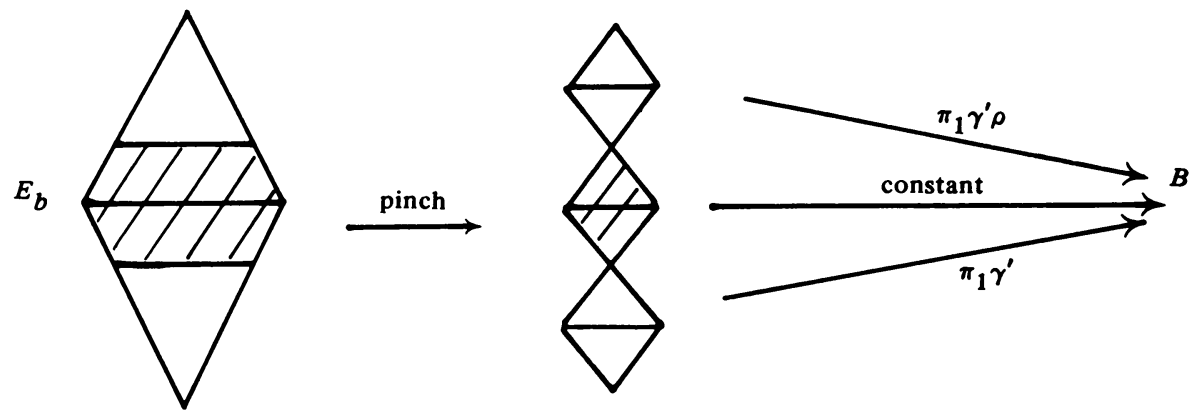

Consequently $\pi_{1} \theta$ is homotopic to $\Sigma E \rightarrow B$ given by

$$
\begin{array}{rlrl}
{[e, t]} & \mapsto \pi_{1} \gamma^{\prime}[e, 2 t+1], & & -1 \leqslant t \leqslant 0, \\
& \mapsto \pi_{1} \gamma^{\prime}[e, 1-2 t], & 0 \leqslant t \leqslant 1 .
\end{array}
$$

But this is homotopic to $\Sigma E \rightarrow B$ given by

$$
[e, t] \mapsto \pi_{1} \gamma^{\prime}[e,-1],
$$

which is $\pi_{1} \gamma^{\prime} \rho s_{2} \pi$. But $\pi_{1} \gamma^{\prime} \rho s_{2} \pi_{2} \simeq \pi_{1} \gamma s_{2} \pi_{2}$.

Since $E_{1}$ is a fibration we can choose $\theta^{\prime} \simeq \theta$ so that the diagram

$$
\begin{array}{ccc}
\sum E & \stackrel{\theta^{\prime}}{\rightarrow} & E_{1} \\
\pi_{2} \downarrow & & \downarrow \pi_{1} \\
B & \stackrel{\pi_{1} \gamma s_{2}}{\rightarrow} & B
\end{array}
$$

commutes. Applying our lemma again, we see that $\pi_{1} \gamma s_{2}$ is a homotopy equivalence. 
4. Fibres. To prove Theorem 2 it suffices to show that the restriction $\phi^{\prime}: S_{2}^{q} \rightarrow S_{1}^{q}$ of $\theta^{\prime}$ to fibres is a homotopy equivalence. In either case we note that

$$
\iota_{1} \phi^{\prime}=\theta^{\prime} \iota_{2} \simeq \theta \iota_{2}=*\left(s_{1} \pi_{1} \gamma^{\prime}, s_{1} \pi_{1} \gamma \rho s_{2} \pi_{2}, \gamma^{\prime} \rho\right) \iota_{2}
$$

and $s_{1} \pi_{1} \gamma \rho s_{2} \pi_{2} \iota_{2}$ is constant.

Case (i). The homomorphism $\left(\iota_{1} \phi^{\prime}\right)_{*}: \pi_{q} S_{2}^{q} \rightarrow \pi_{q} E_{1}$ is

$$
\left(s_{1} \pi_{1} \gamma^{\prime} \iota_{2}\right)_{*}+\left(\gamma^{\prime} \rho \iota_{2}\right)_{*}=\left(s_{1} \pi_{1} \gamma \rho \iota_{2}\right)_{*}+\left(\gamma \rho^{2} \iota_{2}\right)_{*},
$$

which is $\left(\gamma \iota_{2}\right)_{*}$. Now $\gamma$ is a $\phi^{\prime \prime}: S_{2}^{q} \rightarrow S_{1}^{q}$. But the counting argument of $[6, \S 2]$ proves that $\iota_{1 *}: \pi_{q} S_{1}^{q} \rightarrow \pi_{q} E_{1}$ is injective so $\phi^{\prime} \simeq \phi^{\prime \prime}$.

Case (ii). By Proposition $1 E_{1}$ is $\mathbf{Z}$-orientable because $E$ is, and by Theorem $\mathbf{J}$ $\chi\left(E_{1}\right)$ vanishes because $\chi\left(E_{2}\right)$ does. So we have the diagram

$$
\begin{aligned}
& 0 \rightarrow H^{q} B \stackrel{\pi_{1}^{*}}{\rightarrow} H^{q} E_{1} \stackrel{\iota_{1}^{*}}{\rightarrow} H^{q} S_{1}^{q} \rightarrow 0 \\
& \gamma^{*} \downarrow \uparrow \tau^{*} \\
& 0 \rightarrow H^{q} B \stackrel{\pi_{2}^{*}}{\rightarrow} H^{q} E_{2} \stackrel{\iota_{2}^{*}}{\rightarrow} H^{q} S_{2}^{q} \rightarrow 0
\end{aligned}
$$

with exact rows. Here we take coefficients in $\mathbf{Z}$. Now

$$
\iota_{1} \phi^{\prime}=\phi^{\prime} \iota_{2} \simeq \theta \iota_{2}=*\left(s_{1} \pi_{1} \gamma^{\prime}, s_{1} \pi_{1} \gamma \rho s_{2} \pi_{2}, \gamma^{\prime} \rho\right) \iota_{2}
$$

and we note that $s_{1} \pi_{1} \gamma \rho s_{2} \pi_{2} \iota_{2}$ is constant. So $\left(\iota_{1} \phi^{\prime}\right)^{*}: H^{q} E_{1} \rightarrow H^{q} S_{2}^{q}$ is $\left(s_{1} \pi_{1} \gamma^{\prime} \iota_{2}\right)^{*}+$ $\left(\gamma^{\prime} \rho \iota_{2}\right)^{*}$. Since $H^{q}(B ; \mathbf{Z})$ is finite we must have $\left(\pi_{1} \gamma^{\prime} \iota_{2}\right)^{*}$ trivial, so $\left(\iota_{1} \phi^{\prime}\right)^{*}=\left(\gamma^{\prime} \rho \iota_{2}\right)^{*}$ $=\left(\gamma \iota_{2}\right)^{*}=\iota_{2}^{*} \gamma^{*}$, which is surjective. So $\phi^{\prime}$ is a homotopy equivalence and this completes the proof.

\section{APPENDIX. $B=S^{q}$}

Let $B=S^{q}(q>1)$ and let $\tau: E_{1} \rightarrow E_{2}$ be a homotopy equivalence with homotopy inverse $\gamma$. Let $\xi, \xi_{2}$ generate $H^{q} S^{q}, H^{q} S_{2}^{q}$ with coefficients in Z. Let $\left(\pi_{1} \gamma \iota_{2}\right)^{*} \xi=m \xi_{2}$. We say there is no exchange at the set $M$ of primes which divide $m$. Then if $s_{2}$ is a cross-section of the localized fibration $E_{2 M},\left(\pi_{1} \gamma\right)_{M} s_{2}: S_{M}^{q} \rightarrow S_{M}^{q}$ is a homotopy equivalence. This follows from the argument of II, $\$ 2$.

A calculation, using [5, Theorem 7.8] for example, shows that $E_{2}$ is fibre homotopy equivalent to $\sum E$ for some $(q-1)$-spherical fibration $E$. We identify $E_{2 M}$ with $\Sigma\left(E_{M}\right)$ and let $s_{2}$ be the cross-section given by $s_{2}(b)=[e, 1]$ where $\pi_{2 M}(e)=b$. As in III, $\$ 3$ we obtain a commuting diagram

$$
\begin{array}{ccc}
\sum\left(E_{M}\right) & \stackrel{\theta}{\rightarrow} & E_{1 M} \\
\pi_{2 M} \downarrow & & \downarrow \pi_{1 M} \\
B_{M} & \stackrel{\left(\pi_{1} \gamma\right)_{M} s_{2}}{\rightarrow} & B_{M} .
\end{array}
$$

Let $\phi^{\prime}: S_{2 M}^{q} \rightarrow S_{1 M}^{q}$ be the restriction of $\theta^{\prime}$ to fibres. As in III, $\S 4$ we see that $\left(\iota_{1 M} \phi^{\prime}\right)^{*}: H^{q} E_{1 M} \rightarrow H^{q} S_{2 M}^{q}$ is $\left(\gamma \iota_{2}\right)_{M}^{*}+\left(s_{1}\left(\pi_{1} \gamma \iota_{2}\right)_{M}\right)^{*}$, where $s_{1}$ is a cross-section of $E_{1 M}$. Because of the way we chose $M,\left(\pi_{1} \gamma \iota_{2}\right)_{M}^{*}$ is not surjective, so $\phi^{\prime *} \iota_{1 M}^{*}$ is 
surjective. Therefore $\left(\iota_{1 M} \phi^{\prime}\right)^{*}=\phi^{\prime *} \iota_{1 M}^{*}$ is surjective, and $\phi^{\prime}$ is a homotopy equivalence.

Let $N$ be the set of primes which do not divide $m$. We say there is an exchange at $N$. Note that $E_{1 N}, E_{2 N}$ are fibre homotopy trivial. Since $\pi_{q-1} \mathrm{SO}(q+1)$ has order at most 2 when $q$ is odd, we have the following special case of Theorem JW. Note, however, that we do not require $E_{1}$ to be a fibre bundle.

Proposition A.1. Let $E_{2}$ be an orthogonal q-sphere bundle over $S^{q}$ where $q$ is odd. If $E_{1} \simeq E_{2}$ then $E_{1}, E_{2}$ are fibre homotopy equivalent.

Next we consider the case where $q$ is even. We have $\left(\pi_{1} \gamma \iota_{2}\right)^{*} \xi=m \xi_{2}$, and we let $\left(\pi_{1} \gamma d_{2}\right)^{*} \xi=n \xi$, where $s_{2}$ now denotes a cross-section of $E_{2}$. Let $a=\pi_{2}^{*} \xi$ and choose $a_{2} \in H^{q} E_{2}$ as in I, §2 so that $s_{2}^{*} a_{2}=0$ and $\iota_{2}^{*} a_{2}=\xi_{2}$. Then $\gamma^{*} \pi_{1}^{*} \xi=n a+m a_{2}$ so $(m, n)=1$. Since $q$ is even we have $m\left(2 n a \cdot a_{2}+m a_{2}^{2}\right)=\gamma^{*} \pi_{1}^{*} \xi^{2}=0$, so either

(i) $m=0$, or

(ii) $m= \pm 1$, or

(iii) $m= \pm 2$.

In case (i) we may again adapt the proof of Theorem 2, to obtain

Proposition A.2. Let $B=S^{q}$ where $q$ is even. If $E_{1} \simeq E_{2}$ then there is a homotopy equivalence $f: S^{q} \rightarrow S^{q}$ such that $E_{1}, f^{*} E_{2}$ have the same fibre homotopy type.

In this way we recover the remainder of Theorem JW. However we do not require $E_{1}, E_{2}$ to be fibre bundles. We conclude by proving Proposition A.2 in cases (ii) and (iii).

In case (ii) both $E_{1}, E_{2}$ are fibre homotopy trivial. In case (iii) there is a fibre preserving map

$$
\begin{array}{llll}
E_{2} & & \stackrel{\pi_{2} \times \pi_{1} \gamma}{\rightarrow} & \\
& & \pi_{2} \searrow & \\
& & S^{q} \times S^{q}
\end{array}
$$

which is of degree \pm 2 on fibres. Let $\alpha \in \pi_{q-1} G_{1}^{q}$ classify $E_{2}$. Then a calculation using [18 and 19] shows that $\alpha$ has order at most 2. The proof of Proposition A.1 now applies.

\section{REFERENCES}

1. A. K. Bousfield and D. M. Kan, Homotopy Limits, completions and localizations, Lecture Notes in Math., Vol. 304, Springer-Verlag, Berlin and New York, 1972.

2. A. Dold, Partitions of unity in the theory of fibrations, Ann. of Math. (2) 78 (1963), 223-255.

3. P. Hilton and J. Roitberg, On principal $S^{3}$-bundles over spheres, Ann. of Math. (2) 90 (1969), 91-107.

4. S. Y. Husseini, Spherical fibrations, Lecture Notes in Math., Vol. 168, Springer-Verlag, Berlin and New York, 1970, pp. 107-124.

5. I. M. James, On the suspension sequence, Ann. of Math. (2) 65 (1957), 74-107.

6. Which fibre spaces are decomposable?, Indag. Math. 37 (1975), 385-390.

7. I. M. James and J. H. C. Whitehead, Note on fibre spaces, Proc. London Math. Soc. 3 (1954), $129-137$.

8. , The homotopy theory of sphere bundles over spheres, Proc. London Math. Soc. 3 (1954), 196-218. 
9. The homotopy theory of sphere bundles over spheres. II, Proc. London Math. Soc. 3 (1955);

$148-166$

10. W. S. Massey, On the cohomology ring of a sphere bundle, J. Math. Mech. 7 (1958), 265-289.

11. J. W. Milnor and J. D. Stasheff, Characteristic classes, Ann. of Math. Studies, No. 76, Princeton Univ. Press, Princeton, N.J., 1974.

12. G. Mislin, The genus of an H-space, Lecture Notes in Math., Vol. 249, Springer-Verlag, Berlin and New York, 1971, pp. 75-83.

13. J. L. Noakes, Spherical fibrations, Bull. Amer. Math Soc. (N.S.) 3 (1980), 853-856.

14. __ Self-maps of sphere bundles. I, J. Pure Appl. Algebra 10 (1977), 95-99.

15. Unstable J-invariants, Quart. J. Math. Oxford Ser. 27 (1976), 51-57.

16. Flows on fibre bundles, Trans. Amer. Math. Soc. 259 (1980), 629-635.

17. J. D. Stasheff, $A$ classification theorem for fibre spaces, Topology 2 (1963), 239-246.

18. G. W. Whitehead, On products in homotopy groups, Ann. of Math. (2) 47 (1946), 460-475.

19. __ A generalization of the Hopf invariant, Ann. of Math. (2) 51 (1950), 192-237.

Department of Mathematics, University of Western Australia, Nedlands, West Australia 6009, AUSTRALIa 